\title{
Learning Modifies Subsequent Induction of Long-Term Potentiation-Like and Long-Term Depression-Like Plasticity in Human Motor Cortex
}

\author{
Ulf Ziemann, Tihomir V. Iliać, Christian Pauli, Frank Meintzschel, and Diane Ruge \\ Motor Cortex Laboratory, Clinic of Neurology, Johann Wolfgang Goethe-University, D-60528 Frankfurt am Main, Germany
}

\begin{abstract}
Learning may alter rapidly the output organization of adult motor cortex. It is a long-held hypothesis that modification of synaptic strength along cortical horizontal connections through long-term potentiation (LTP) and long-term depression (LTD) forms one important mechanism for learning-induced cortical plasticity. Strong evidence in favor of this hypothesis was provided for rat primary motor cortex (M1) by showing that motor learning reduced subsequent LTP but increased LTD. Whether a similar relationship exists in humans is unknown. Here, we induced LTP-like and LTD-like plasticity in the intact human M1 by an established paired associative stimulation (PAS) protocol. PAS consisted of 200 pairs of electrical stimulation of the right median nerve, followed by focal transcranial magnetic stimulation of the hand area of the left M1 at an interval equaling the individual N20 latency of the median nerve somatosensory-evoked cortical potential $\left(\mathrm{PAS}_{\mathrm{N} 20}\right)$ or $\mathrm{N} 20-5 \mathrm{msec}\left(\mathrm{PAS}_{\mathrm{N} 20-5}\right)$. $\mathrm{PAS}_{\mathrm{N} 20}$ induced reproducibly a LTP-like long-lasting $(>30 \mathrm{~min})$ increase in motor-evoked potentials from the left $\mathrm{M} 1$ to a thumb abductor muscle of the right hand, whereas PAS $\mathrm{N} 20-5_{5}$ induced a LTD-like decrease. Repeated fastest possible thumb abduction movements resulted in learning, defined by an increase in maximum peak acceleration of the practiced movements, and prevented subsequent $\mathrm{PAS}_{\mathrm{N} 20}$-induced LTP-like plasticity but enhanced subsequent $\mathrm{PAS}_{\mathrm{N} 20-5}$-induced LTDlike plasticity. The same number of repeated slow thumb abduction movements did not result in learning and had no effects on PASinduced plasticity. Findings support the view that learning in human M1 occurs through LTP-like mechanisms.
\end{abstract}

Key words: learning; long-term potentiation (LTP); associative plasticity; motor-evoked potential; transcranial magnetic stimulation; human motor cortex

\section{Introduction}

Learning may alter rapidly the output organization of $\mathrm{M} 1$ in adult humans (Pascual-Leone et al., 1995; Classen et al., 1998; Liepert et al., 1999; Muellbacher et al., 2001, 2002), nonhuman primates (Nudo et al., 1996), and rodents (Kleim et al., 1998). One important hypothesis on the mechanisms of learning-induced cortical plasticity is that synaptic strength of cortical horizontal connections is modified through long-term potentiation (LTP) and long-term depression (LTD) (Donoghue et al., 1996; Sanes and Donoghue, 2000).

Strong evidence in favor of this hypothesis was provided recently in rat M1 (Rioult-Pedotti et al., 1998, 2000). Forelimb skill learning was associated with an increase in the synaptic strength of horizontal cortical connections of the forelimb area of the trained M1 but not the untrained opposite M1 or the hindlimb area of the trained cortex. The amount of LTP that could be induced in the trained $\mathrm{M} 1$ was less than in the untrained cortex or in the M1 of untrained control animals. This strongly suggested that skill learning occurred, at least in part, through LTP. Addi-

\footnotetext{
Received Nov. 10, 2003; revised Jan. 6, 2004; accepted Jan. 6, 2004. T.V.I. was a fellow of the Alexander von Humboldt Foundation.

Correspondence should be addressed to Dr. UlfZiemann, Clinic of Neurology, Johann Wolfgang Goethe University of Frankfurt, Theodor-Stern-Kai 7, D-60590 Frankfurt am Main, Germany. E-mail: u.ziemann@em.uni-frankfurt.de. DOI:10.1523/JNEUROSCI.5016-03.2004

Copyright $\odot 2004$ Society for Neuroscience $\quad$ 0270-6474/04/241666-07\$15.00/0
}

tional experiments showed that the synaptic modification range in the trained M1 remained unchanged: skill learning prevented LTP whereas the induction of LTD was enhanced (Rioult-Pedotti et al., 2000).

It is unknown whether a similar relationship between learning and LTP exists in the human cortex. Therefore, we tested here to which extent motor learning modifies subsequent induction of LTP/D-like plasticity in the human M1.

MP was adopted from an established learning protocol (Muellbacher et al., 2001) and consisted of repeated fastest possible thumb abduction movements. Learning manifests as an increase in maximum peak acceleration of the practiced movements, associated with a rapid change in M1 excitability, as measured by an increase in motor-evoked potential (MEP) amplitude in the prime mover muscle of the task (Muellbacher et al., 2001).

Plasticity in M1 was induced according to a paired associative stimulation (PAS) protocol (Stefan et al., 2000; Wolters et al., 2003). PAS consisted of 200 pairs of electrical stimulation of the right median nerve, followed by focal transcranial magnetic stimulation (fTMS) of the hand area of the left M1 at an interval equaling the individual N20 latency of the median nerve somatosensory-evoked cortical potential $\left(\mathrm{PAS}_{\mathrm{N} 20}\right)$ or $\mathrm{N} 20-5$ msec $\left(\mathrm{PAS}_{\mathrm{N} 20-5}\right)$. $\mathrm{PAS}_{\mathrm{N} 20}$ leads to an LTP-like long-lasting increase in MEP amplitude of a median nerve innervated hand 


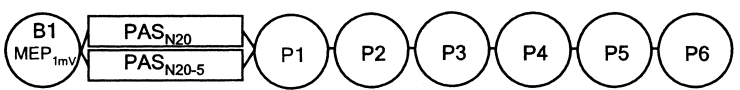

MP

alone
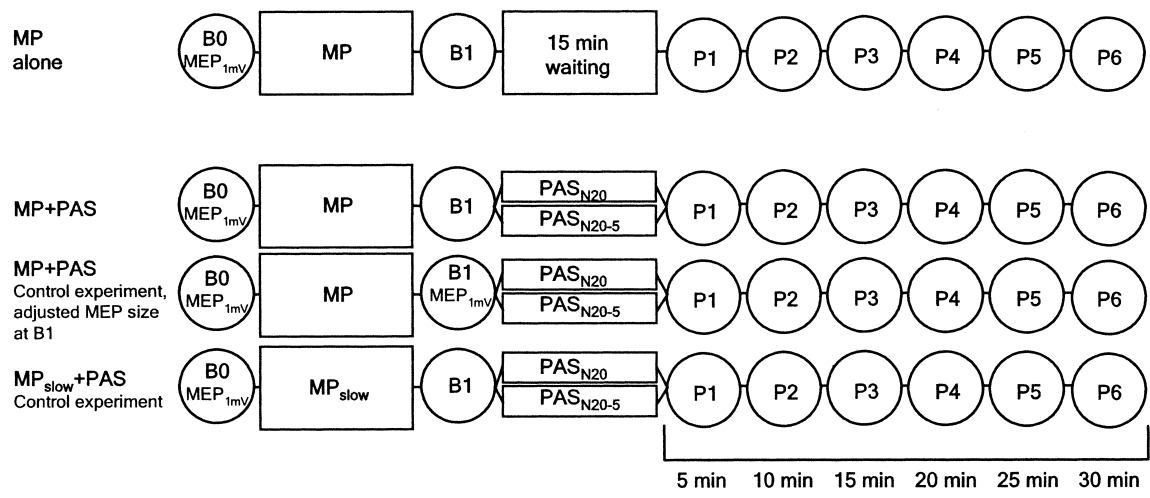

Figure 1. Timeline of experiments (for details, see Materials and Methods).

plitude in the resting APB when given without the preceding median nerve stimulus. MEPs were recorded from the APB by surface electromyography (EMG), using $\mathrm{Ag}-\mathrm{AgCl}$ cup electrodes in a belly-tendon montage. The EMG raw signal was amplified and filtered $(0.05-2$ $\mathrm{kHz}$; Counterpoint Mk2 electromyograph; Dantec, Skovlunde, Denmark), digitized [analog-to-digital (A/D) rate, $5 \mathrm{kHz}$; CED Micro 1401; Cambridge Electronic Design, Cambridge, UK), and fed into an IBM-compatible Pentium 5 laboratory computer for online visual display and offline analysis.

Two interstimulus intervals between the median nerve stimulus and fTMS were tested. One equaled the individual N20 cortical component of the median nerve somatosensory-evoked potential $\left(\mathrm{PAS}_{\mathrm{N} 20}\right)$, and the other interval was shorter by $5 \mathrm{msec}\left(\mathrm{PAS}_{\mathrm{N} 20-5}\right)$. These intervals were selected because previous experiments showed that $\mathrm{PAS}_{\mathrm{N} 20}$ results consistently in a long-lasting LTP-like MEP increase whereas

muscle, whereas $\mathrm{PAS}_{\mathrm{N} 20-5}$ leads to a LTD-like decrease (Stefan et al., 2000, 2002; Wolters et al., 2003) (our unpublished observations). These changes in MEP amplitude are called LTP/D like because they show similar characteristics as LTP/D (Bliss and Collingridge, 1993), in particular duration ( $>60 \mathrm{~min}$ ), input specificity, and dependence on NMDA receptor activation (Stefan et al., 2000, 2002; Wolters et al., 2003). Still, this concept is based on analogies, and it cannot be proven definitely that LTP/ D-like plasticity is the same as LTP/D.

The specific hypothesis tested here was that motor learning prevents subsequent LTP-like plasticity but enhances subsequent LTD-like plasticity.

\section{Materials and Methods}

\section{Subjects}

Twelve naive right-handed healthy subjects (24-42 years old; four females) participated in the experiments. Nobody had a history of neurological disease or was on CNS-active drugs at the time of the experiments. All subjects gave written informed consent. The experiments conformed to the Declaration of Helsinki and were approved by the local ethics committee.

\section{Timeline of experiments (Fig. 1)}

Each subject participated in five experiments of fixed order, at least 1 week apart from each other: the first two sessions applied PAS alone (PAS alone), the third session tested the effects of motor practice alone (MP alone), and the forth and fifth sessions assessed the interactions between $\mathrm{MP}$ and PAS (MP+PAS). Finally, six subjects participated in a control experiment that assessed the interactions between an MP without learning $\left(\mathrm{MP}_{\text {slow }}\right)$ and PAS.

\section{Paired associative stimulation}

PAS consisted of 200 electrical stimuli of the right median nerve at the wrist paired with fTMS of a site over of the hand area of the left M1 optimal for eliciting MEP in the right abductor pollicis brevis (APB) muscle. The rate of paired stimulation was $0.25 \mathrm{~Hz}$. Electrical stimulation was applied through a bipolar electrode (cathode proximal), using constant current square wave pulses (duration, $1 \mathrm{msec}$ ) at an intensity of three times the perceptual threshold. fTMS was delivered through a figure-eight coil (diameter of each wing, $70 \mathrm{~mm}$ ) connected to a Magstim 200 magnetic stimulator with a monophasic current waveform (The Magstim Company, Carmarthenshire, Wales, UK). The coil was held tangential to the scalp with the handle pointing backward and $45^{\circ}$ away from the midline. This is the optimal orientation for activating the corticospinal system transsynaptically via horizontal cortical connections (Sakai et al., 1997). In most experiments (see below), the intensity of fTMS was adjusted to produce an MEP of $\sim 1 \mathrm{mV}$ in peak-to-peak am-
$\mathrm{PAS}_{\mathrm{N} 20-5}$ leads to a LTD-like MEP decrease (Stefan et al., 2000, 2002; Wolters et al., 2003) (our unpublished observations). With $\mathrm{PAS}_{\mathrm{N} 20}$, the associative stimuli activate M1 approximately simultaneously. In contrast, with $\mathrm{PAS}_{\mathrm{N} 20-5}, \mathrm{M} 1$ activation by fTMS leads the activation by median nerve stimulation by $\sim 5 \mathrm{msec}$. Each subject was assigned randomly with a $50 \%$ chance to $\mathrm{PAS}_{\mathrm{N} 20}$ (LTP group) or $\mathrm{PAS}_{\mathrm{N} 20-5}$ (LTD group). This assignment was blinded toward experimenter and subjects. Each subject was tested twice for the selected PAS condition to assess the reproducibility of PAS effects (PAS alone experiments).

\section{Quantification of PAS effects}

MEP amplitudes were measured to assess changes in the output organization of the left M1 induced by PAS. MEP amplitude reflects synaptic excitability in M1, which is regulated through various inhibitory and excitatory neurotransmitter systems (Boroojerdi et al., 2001). In addition, MEP amplitude may be affected by excitability changes of spinal motoneurons, but previous experiments provided strong evidence that changes in MEP amplitude induced by PAS occur mainly or exclusively at the cortical level (Stefan et al., 2000; Ridding and Taylor, 2001; Wolters et al., 2003).

MEPs were elicited in the relaxed APB of the right hand by fTMS applied to the hand area of the left M1 (see above). Relaxation of the APB was monitored audio-visually with high-gain EMG (50 $\mu \mathrm{V} /$ division). Trials contaminated with voluntary EMG activity were discarded from analysis. The data of one subject in the LTD group had to be removed completely from analysis because this subject was not able to fully relax the APB in the experiments testing the interactions between MP and PAS. The time points of MEP measurements are shown in Figure 1 (B0, B1, $\mathrm{P} 1-\mathrm{P} 6)$. At time point B1 (PAS alone experiments) or B0 (all other experiments), fTMS intensity was adjusted to elicit MEPs of, on average, 1 $\mathrm{mV}$ in peak-to-peak amplitude $\left(\mathrm{MEP}_{1 \mathrm{mV}}\right)$. In addition, fTMS intensity was readjusted at time point $\mathrm{B} 1$ in one of the two MP+PAS sessions to reinstall $\mathrm{MEP}_{1 \mathrm{mV}}$ after any MP-induced increase in MEP amplitude (Fig. 1). This control experiment allowed a direct comparison of the PAS effects with and without prior MP. At each time point of MEP measurements, 20 MEPs were obtained, using a mean intertrial interval of $10 \mathrm{sec}$ with a random intertrial interval variation of $25 \%$. For each subject and time point, the single-trial peak-to-peak MEP amplitudes were averaged and normalized to the MEP amplitude measured at B1 (compare Figs. $1-6)$.

\section{Motor practice}

Subjects were seated in a comfortable chair. Their right arm was adducted in the shoulder, $90^{\circ}$ flexed in the elbow, and the semi-pronated forearm rested on a plate. Forearm, wrist, and fingers II-V were fixed in a cast, leaving the thumb free for movements in all directions. The learning task was adopted from Muellbacher et al. (2001) and consisted of 
fastest thumb abduction movements of the right hand for $30 \mathrm{~min}$, paced by a brief $1000 \mathrm{~Hz}$ tone at a rate of $0.5 \mathrm{~Hz}$. Acceleration of the thumb movements was measured using two uniaxial accelerometers (model 2256A-100; voltage sensitivity, $100 \mathrm{mV} / \mathrm{g}$; Endevco, San Juan Capistrano, $\mathrm{CA})$. The accelerometers were mounted on the thumb in orthogonal planes to detect acceleration in the abduction-adduction and extensionflexion axes, respectively. The raw signal was amplified (model 133 signal conditioner; Endevco), digitized (A/D rate, $5 \mathrm{kHz}$; CED Micro 1401) and fed into the laboratory computer for online visual display and offline analysis. Subjects were continuously encouraged by the experimenter to make ballistic and isolated thumb abduction movements that led to as high as possible acceleration of the first peak in the direction of abduction but as low as possible acceleration along the extension-flexion axis.

\section{Quantification of the learning effect}

Previous experiments showed that repeated fastest finger movements lead rapidly to an increase in peak acceleration and force of the fingers involved in the task (Muellbacher et al., 2001, 2002). We quantified the learning effect by comparing the mean first peak acceleration of 10 externally paced $(1000 \mathrm{~Hz}$ tone at a rate $0.1 \mathrm{~Hz})$ fastest possible thumb abduction movements measured immediately before and after MP. In addition, repeated fastest finger movements resulted in an increase in MEP amplitude in the prime mover muscle (Muellbacher et al., 2001, 2002). We quantified this change in motor excitability by measuring the MEP amplitude in the resting APB immediately before (B0) and after (B1) MP. Stimulus intensity was adjusted to result in peak-to-peak MEP amplitudes of, on average, $1 \mathrm{mV}$ at $\mathrm{B} 0\left(\mathrm{MEP}_{1 \mathrm{mV}}\right)$ (Fig. 1$)$. In addition, long-term effects of MP on motor excitability were evaluated by comparing MEP amplitudes over a period of $30 \mathrm{~min}$ after the $15 \mathrm{~min}$ waiting period (P1-P6) (Fig. 1) with the MEP amplitude immediately after MP (B1).

\section{Control experiment $\left(M P_{\text {slow }}\right)$}

To test whether any interactions between MP and the PAS effects were specific to motor learning, a control experiment was designed that used the same amount of MP but without learning. This form of MP was also adopted from a previously described protocol (Muellbacher et al., 2001) and consisted of slow, ramp-like thumb abduction movements ( $\left.\mathrm{MP}_{\text {slow }}\right)$. Subjects were continuously encouraged by the experimenter to keep the duration of the thumb abduction movements within 500-1000 msec. All other conditions of the practice task (total number of movements, 900; externally paced movement rate, $0.5 \mathrm{~Hz}$ ) matched the MP protocol (see above). $\mathrm{MP}_{\text {slow }}$ does not result in motor learning when defined by an increase in maximum peak acceleration of the trained movements and is not associated with an increase in MEP amplitude in the prime mover muscles of the task (Muellbacher et al., 2001).

\section{Data analysis and statistics}

PAS alone. Changes in MEP amplitude induced by PAS were analyzed by two-tailed paired $t$ tests to compare MEP amplitude after PAS (average of time points $\mathrm{P} 1-\mathrm{P} 6$ ) with MEP amplitude before PAS (time point B1). Tests were run separately for the LTP and LTD groups and for sessions 1 and 2. In addition, changes in MEP amplitude were expressed by the ratio of MEP amplitude at any given time point over the MEP amplitude at time point B1. To test for the effects of session and group, a three-way ANOVA was run with the within-subject effects time (P1-P6) and session (sessions 1 and 2) and the between-subject effect group (LTP, LTD).

MP alone. Changes in peak acceleration and MEP amplitude induced by MP were analyzed separately by a two-way ANOVA with time (B1 vs B0) as within-subject effect and group (LTP vs LTD) as between-subject effect. Another two-way ANOVA evaluated changes in MEP amplitude over the $30 \mathrm{~min}$ following the $15 \mathrm{~min}$ waiting period compared with MEP amplitude directly after MP (Fig. 1). In this ANOVA, time (P1-P6 vs B1) was the within-subject effect and group (LTP vs LTD) was the betweensubject effect.

$M P$ followed by PAS (MP+PAS). The effects of MP on PAS-induced changes in MEP amplitude were tested separately for the LTP and LTD groups, using a two-way ANOVA with the within-subject effects experimental condition (grand average from the two sessions of PAS alone vs $\mathrm{MP}+\mathrm{PAS})$ and time (P1-P6). This analysis was repeated for the control

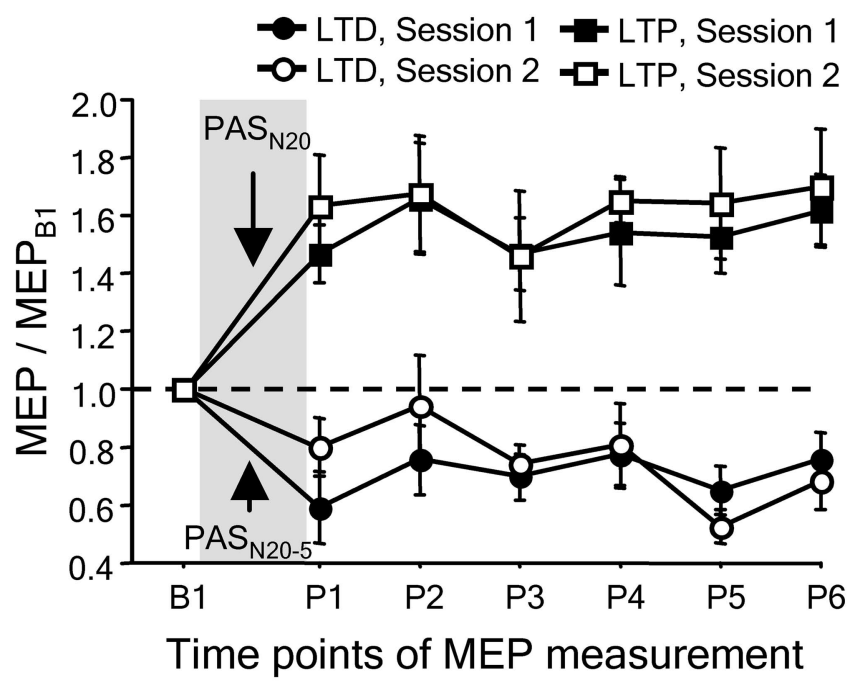

Figure 2. Lasting increase of MEP amplitude in the resting APB muscle induced by PAS $\left(\mathrm{PAS}_{\mathrm{N2} 2}\right.$; squares) and MEP decrease induced by $\mathrm{PAS}_{\mathrm{N2} 2-5}$ (circles). The times of MEP testing are denoted on the $x$-axis (compare Fig. 1). MEPs are normalized to MEP amplitude measured at B1. Each subject was tested twice (session 1, black symbols; session 2, white symbols). All data are the means \pm 1 SEM from six subjects.

experiment in which fTMS intensity was adjusted (reinstallation of $\mathrm{MEP}_{1 \mathrm{mV}}$ at $\mathrm{B} 1$ ) to compensate for any MP-induced increase in MEP amplitude. In addition, the MP-induced increases in peak acceleration and MEP amplitude were compared between the LTP and LTD groups using a two-tailed $t$ test for independent samples. These tests were important to exclude differences in learning performance before PAS between the two experimental groups.

$M P_{\text {slow }}+$ PAS. The effects of $\mathrm{MP}_{\text {slow }}$ on PAS-induced changes in MEP amplitude (normalized to B1) were tested with a three-way ANOVA with the within-subject effects experimental condition (grand average from the two sessions of PAS alone vs $\left.\mathrm{MP}_{\text {slow }}+\mathrm{PAS}\right)$ and time (P1-P6) and the between-subject effect group (LTP vs LTD).

In all tests, results were considered significant at a level of $p<0.05$.

\section{Results}

\section{Effects of $\mathrm{PAS}_{\mathrm{N} 20}$ alone and $\mathrm{PAS}_{\mathrm{N} 20-5}$ alone on}

MEP amplitude

In the LTP group, PAS $_{\mathrm{N} 20}$ resulted in an increase in MEP amplitude in the APB in both sessions (session 1: $0.88 \pm 0.24 \mathrm{mV} \rightarrow$ $1.36 \pm 0.39 \mathrm{mV}, T=4.78, p=0.005$; session $2: 0.92 \pm 0.24$ $\mathrm{mV} \rightarrow 1.47 \pm 0.44 \mathrm{mV}, T=3.63, p=0.015)$ that lasted for at least 30 min (Fig. 2, squares). In the LTD group, $\mathrm{PAS}_{\mathrm{N} 20-5}$ resulted in a decrease of MEP amplitude in both sessions (session 1: $1.03 \pm 0.32 \mathrm{mV} \rightarrow 0.73 \pm 0.33 \mathrm{mV}, T=-3.30, p=0.021$; session 2: $1.01 \pm 0.30 \mathrm{mV} \rightarrow 0.75 \pm 0.17 \mathrm{mV}, T=-3.98, p=$ 0.011 ) that also lasted for at least $30 \mathrm{~min}$ (Fig. 2, circles). The difference in MEP modulation between the LTP and LTD groups was highly significant $\left(F_{(1,10)}=54.5 ; p<0.0001\right)$, whereas there was no significant effect of session $\left(F_{(1,10)}=0.99 ; p=0.34\right)$ or the interaction of session with group $\left(F_{(1,10)}=0.08 ; p=0.77\right)$.

Effects of MP alone on peak acceleration and MEP amplitude MP resulted in a significant increase in the peak acceleration of the fastest possible voluntary thumb abduction movements from $8.44 \pm 4.83$ to $13.02 \pm 8.42 \mathrm{~m} / \mathrm{sec}^{2}\left(F_{(1,9)}=10.03 ; p=0.011\right)$ without differences between the LTP and LTD groups $\left(F_{(1,9)}=\right.$ $0.32 ; p=0.59)$ and no interaction between time and group $\left(F_{(1,9)}=0.01 ; p=0.98\right)$. The magnitude of the mean increase in peak acceleration of $52.7 \pm 48.2 \%$ (LTP group, $62.4 \pm 60.9 \%$; 


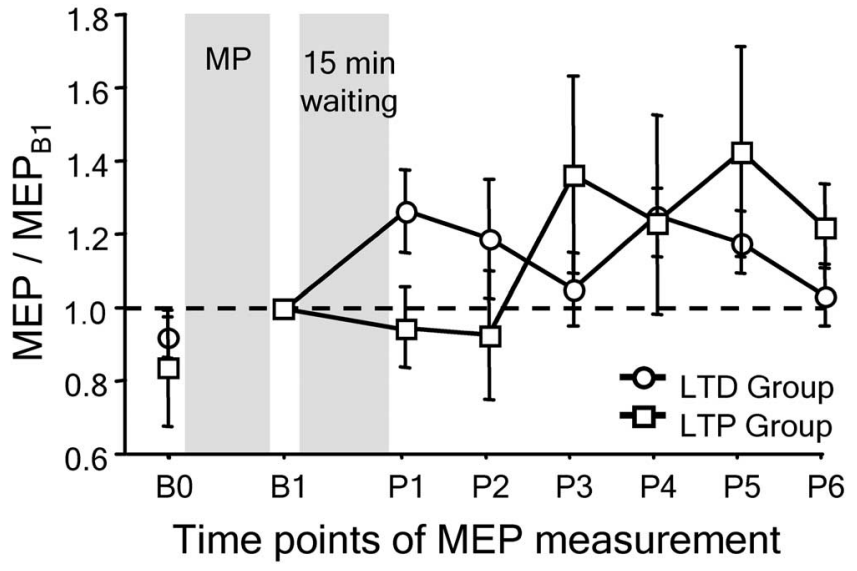

Figure 3. Changes in MEP amplitude induced by MP alone in the LTP (squares) and LTD (circles) groups. Otherwise, arrangement and conventions are as in Figure 2.

LTD group, $40.9 \pm 29.1 \%$ ) is consistent with previous MP experiments (Muellbacher et al., 2001, 2002).

In addition, comparison of the time points immediately before (B0) and after (B1) (Figs. 1 and 3) MP revealed a significant increase in MEP amplitude in the APB from $1.04 \pm 0.23$ to $1.32 \pm$ $0.40 \mathrm{mV}\left(F_{(1,9)}=10.39 ; p=0.010\right)$ without differences between the LTP and LTD groups $\left(F_{(1,9)}=0.30 ; p=0.60\right)$ and no significant interaction between time and group $\left(F_{(1,9)}=4.68 ; p=\right.$ $0.06)$. The magnitude of the mean increase in MEP amplitude of $27.4 \pm 28.5 \%$ (LTP group, $41.4 \pm 30.3 \%$; LTD group, $10.6 \pm$ $15.5 \%$ ) is consistent with the MEP increase reported in previous MP experiments (Muellbacher et al., 2001).

The comparison of MEP amplitude averaged over the $30 \mathrm{~min}$ following the 15 min waiting period (P1-P6) with MEP amplitude measured immediately after MP but before the waiting period (B1) (Fig. 1) revealed an additional nonsignificant increase from $1.32 \pm 0.40$ to $1.48 \pm 0.35 \mathrm{mV}\left(F_{(1,9)}=2.47 ; p=0.15\right)$ without differences between the LTP and LTD groups $\left(F_{(1,9)}=\right.$ $1.63 ; p=0.23$ ) (Fig. 3).

In summary, the MP alone experiments showed that significant learning occurred (increase in peak acceleration). This was associated with a significant increase in motor excitability (increase in MEP amplitude). These effects were not different between the LTP and LTD groups.

\section{Interactions between MP and PAS}

The PAS alone and MP alone experiments fulfilled two important preconditions to assess the interactions of motor learning with PAS-induced plasticity: (1) the two sessions of PAS alone resulted in a highly reproducible MEP increase in the LTP group and an MEP decrease in the LTD group; and (2) motor learning and the associated increase in motor excitability were not different between the LTP and LTD groups.

The interactions of MP with $\mathrm{PAS}_{\mathrm{N} 20}$ and $\mathrm{PAS}_{\mathrm{N} 20-5}$ were greatly different. In the LTP group, the highly reproducible and long-lasting MEP increase induced by the two $\mathrm{PAS}_{\mathrm{N} 20}$ alone experiments (grand average data) (Fig. 4, black squares) was abolished when motor learning immediately preceded $\mathrm{PAS}_{\mathrm{N} 20}$ (Fig. 4, gray squares). The difference between the two curves was highly significant $\left(F_{(1,5)}=25.5 ; p=0.004\right)$. There was even a trend toward a depression of MEP amplitude if MP preceded $\mathrm{PAS}_{\mathrm{N} 20}$, but MEP amplitude was at no time (P1-P6) significantly smaller than MEP amplitude at B1 ( $t$ tests corrected for multiple comparisons; $p>0.05)$. In contrast, in the LTD group, the highly

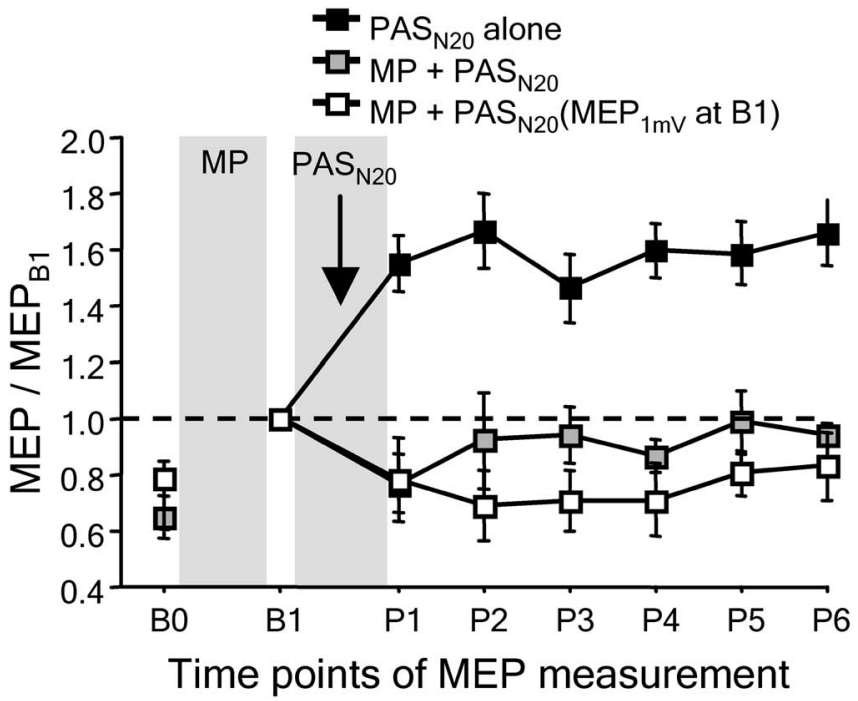

Figure 4. Interactions between $M P$ and $P A S_{\mathrm{N} 20}$. Black squares indicate the lasting increase in MEP amplitude induced by $\mathrm{PAS}_{\mathrm{N} 20}$ alone (average of $\mathrm{PAS}_{\mathrm{N} 20}$ sessions 1 and 2 in Fig. 2). Gray and white squares show the changes in MEP amplitude if $\mathrm{PAS}_{\mathrm{N} 20}$ was preceded by MP associated with learning. White squares refer to the control experiment in which any increase in MEP amplitude induced by MP was compensated for by reduction in fTMS intensity (reinstallation of $\mathrm{MEP}_{1 \mathrm{mv}}$ at B1). Otherwise, arrangement and conventions are as in Figure 2. Note that motor learning prevented the lasting increase in MEP amplitude induced by $\mathrm{PAS}_{\mathrm{N} 20}$ alone.

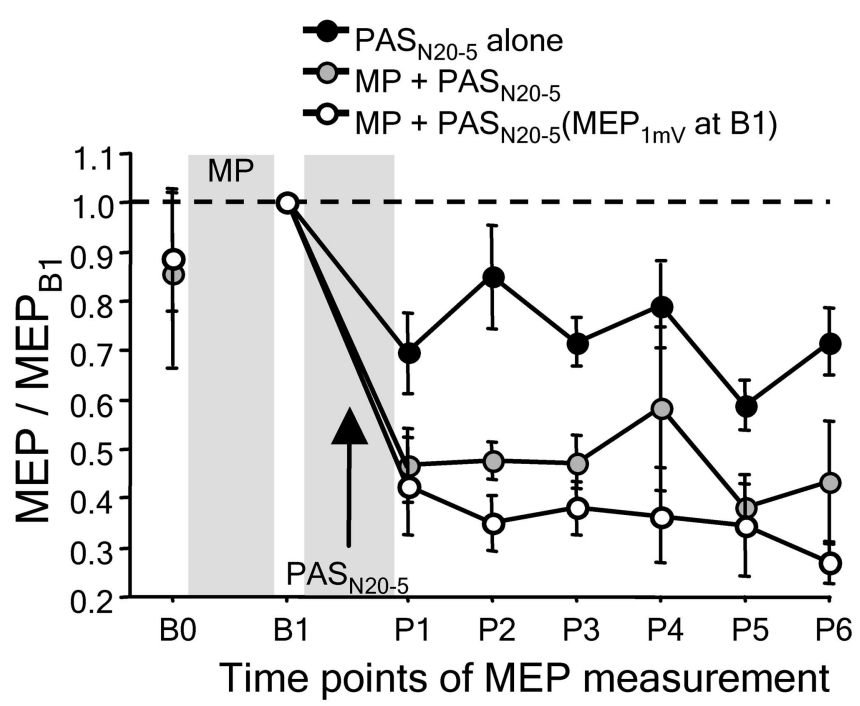

Figure 5. Interactions between $\mathrm{MP}$ and $\mathrm{PAS}_{\mathrm{N} 20-5}$. Arrangement and conventions are as in Figure 4. Note that motor learning enhanced the lasting decrease in MEP amplitude induced by $\mathrm{PAS}_{\mathrm{N} 20-5}$ alone.

reproducible and long-lasting $\mathrm{MEP}$ decrease induced by the two $\mathrm{PAS}_{\mathrm{N} 20-5}$ alone experiments (grand average data) (Fig. 5, black circles) was enhanced if motor learning immediately preceded PAS $_{\mathrm{N} 20-5}$ (Fig. 5, gray circles) $\left(F_{(1,4)}=7.97 ; p=0.048\right)$.

This marked difference between groups in the interaction of motor learning with $\mathrm{PAS}_{\mathrm{N} 20}$ and $\mathrm{PAS}_{\mathrm{N} 20-5}$ was not caused by differences in the learning performance or the learningassociated increase in motor excitability. MP resulted in a significant increase in peak acceleration of fastest voluntary thumb abduction movements from $6.55 \pm 2.55$ to $9.21 \pm 4.59 \mathrm{~m} / \mathrm{sec}^{2}$ $\left(F_{(1,9)}=5.28 ; p=0.047\right)$, which was not different between the LTP and LTD groups, although there was a trend for a stronger learning effect in the LTP group $\left(F_{(1,9)}=5.07 ; p=0.051\right.$ ) (Table 
Table 1. Peak acceleration $(A C C)\left(\mathrm{m} / \mathrm{sec}^{2}\right)$ and MEP amplitude $(\mathrm{mV})$ before and after motor learning

\begin{tabular}{|c|c|c|c|c|c|}
\hline & \multicolumn{2}{|l|}{ LTP group } & \multicolumn{2}{|l|}{ LTD group } & \multirow[b]{2}{*}{$p$} \\
\hline & BO & B1 & BO & B1 & \\
\hline Peak ACC & $7.71 \pm 2.9$ & $11.42 \pm 4.26$ & $5.17 \pm 1.10$ & $6.55 \pm 3.71$ & $\mathrm{~B} 0,0.10 ; \mathrm{B} 1,0.08$ \\
\hline Peak ACC (B1/B0) & $1.56 \pm 0.65$ & & $1.23 \pm 0.58$ & & 0.41 \\
\hline MEP & $0.94 \pm 0.21$ & $1.52 \pm 0.46$ & $1.16 \pm 0.22$ & $1.42 \pm 0.28$ & $B 0,0.11 ; B 1,0.67$ \\
\hline $\operatorname{MEP}(\mathrm{B} 1 / \mathrm{B} 0)$ & $1.66 \pm 0.58$ & & $1.26 \pm 0.38$ & & 0.22 \\
\hline
\end{tabular}

Values are means \pm SD ( $n=6, \mathrm{LTP}$ group; $n=5, \mathrm{LTD}$ group). $p$ values indicate comparisons between the LTP and LTD groups (unpaired $t$ test).

1). A comparison of MEP amplitude between the time points immediately before (B0) and after MP (B1) revealed that motor learning was associated with a significant increase in MEP amplitude from $1.04 \pm 0.24$ to $1.49 \pm 0.36 \mathrm{mV}\left(F_{(1,9)}=13.16 ; p=\right.$ 0.0055), which was not different between the LTP and LTD groups $\left(F_{(1,9)}=0.28 ; p=0.61\right)$ (Table 1$)$.

Finally, the effects within the LTP and LTD groups could not be attributed to the learning-induced increase in MEP amplitude because the control experiment with lowered intensity of fTMS to correct for this increase (reinstallation of $\mathrm{MEP}_{1 \mathrm{mV}}$ at B1) resulted in a very similar prevention of $\mathrm{PAS}_{\mathrm{N} 20}$-induced plasticity in the LTP group and enhancement of $\mathrm{PAS}_{\mathrm{N} 20-5}$-induced plasticity in the LTD group (Figs. 4, 5, white symbols) compared with the experiments without adjustment of fTMS intensity (Figs. 4, 5, gray symbols). In the LTP group, $\mathrm{MP}+\mathrm{PAS}_{\mathrm{N} 20}$ with adjusted fTMS intensity was highly significantly different from $\mathrm{PAS}_{\mathrm{N} 20}$ alone $(p<0.0001)$, whereas it was not different from $\mathrm{MP}+\mathrm{PAS}_{\mathrm{N} 20}$ without adjusted fTMS intensity $(p=0.27)$. Similarly, in the LTD group, MP+ $\mathrm{PAS}_{\mathrm{N} 20-5}$ with adjusted fTMS intensity was significantly different from $\mathrm{PAS}_{\mathrm{N} 20-5}$ alone $(p=$ 0.0052 ), whereas it was not different from $\mathrm{MP}+\mathrm{PAS}_{\mathrm{N} 20-5}$ without adjusted fTMS intensity $(p=0.26)$.

\section{Control experiment: interactions between $\mathrm{MP}_{\text {slow }}$ and PAS}

$\mathrm{MP}_{\text {slow }}$ did not result in a significant change in the peak acceleration of fastest possible thumb abduction movements (LTP group B0: $6.3 \pm 3.2 \mathrm{~m} / \mathrm{sec}^{2}, \mathrm{~B} 1: 5.9 \pm 1.7 \mathrm{~m} / \mathrm{sec}^{2}$; LTD group B0: $\left.6.7 \pm 1.6 \mathrm{~m} / \mathrm{sec}^{2}, \mathrm{~B} 1: 6.3 \pm 0.8 \mathrm{~m} / \mathrm{sec}^{2}\right)$. Also, $\mathrm{MP}_{\text {slow }}$ did not lead to a significant change in MEP amplitude in the APB (LTP group B0: $0.82 \pm 0.17 \mathrm{mV}, \mathrm{B} 1: 0.65 \pm 0.14 \mathrm{mV}$; LTD group B0: $1.41 \pm$ $0.36 \mathrm{mV}, \mathrm{B} 1: 1.36 \pm 0.57 \mathrm{mV})$. Therefore, $\mathrm{MP}_{\text {slow }}$ can be considered as a valid control task that did not result in motor learning or a change in motor excitability.

The ANOVA revealed no effect of the within-subject effect of experimental condition $\left(F_{(1,4)}=0.001 ; p=0.98\right)$, its interaction with time $\left(F_{(5,4)}=0.37 ; p=0.86\right)$, or its interaction with group $\left(F_{(1,4)}=0.59 ; p=0.49\right)$. In contrast, the between-subject effect of group was highly significant $\left(F_{(1,4)}=97.0 ; p=0.0006\right)$. Therefore, the main result of this control experiment is that $\mathrm{MP}_{\text {slow }}$ did not affect the long-lasting increase in MEP amplitude induced by $\mathrm{PAS}_{\mathrm{N} 20}$ alone (Fig. 6, gray vs black squares) or the long-lasting decrease in MEP amplitude induced by $\mathrm{PAS}_{\mathrm{N} 20-5}$ alone (Fig. 6, gray vs black circles).

\section{Discussion}

\section{Associative LTP/D-like plasticity in human motor cortex}

Previous studies showed that the changes in MEP amplitude induced by PAS occur in M1. Concomitant excitability changes in the spinal cord were excluded by electrical brainstem stimulation and measurement of F wave amplitudes (Stefan et al., 2000; Wolters et al., 2003). The increase in MEP amplitude was associated with an increased excitability of excitatory interneurons in M1 (Ridding and Taylor, 2001), whereas intracortical inhibition

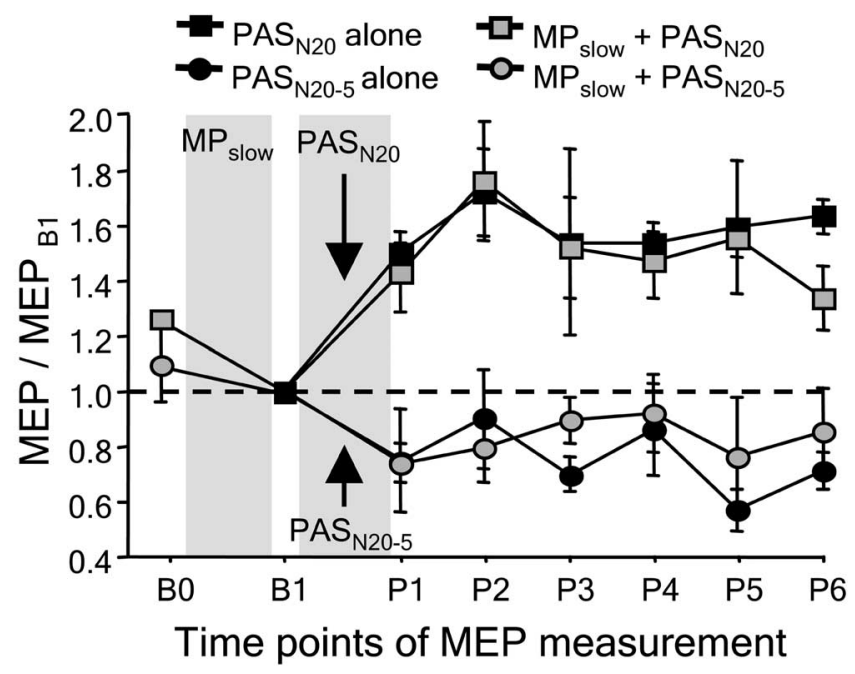

Figure 6. Interactions of $\mathrm{MP}_{\text {slow }}$ with $\mathrm{PAS}_{\mathrm{N} 20}$ and $\mathrm{PAS}_{\mathrm{N} 20-5}$. Squares show normalized MEP amplitudes in the $\mathrm{PAS}_{\mathrm{N} 20}$ alone (black) and $\mathrm{MP}_{\text {slow }}+\mathrm{PAS}_{\mathrm{N} 20}$ (gray) conditions, and circles show MEP amplitudes in the $\mathrm{PAS}_{\mathrm{N} 20-5}$ alone (black) and $\mathrm{MP}_{\text {slow }}+\mathrm{PAS}_{\mathrm{N} 20-5}$ (gray) conditions. Note that $\mathrm{MP}_{\text {slow }}$ had no effect on either the lasting increase in MEP amplitude induced by $\mathrm{PAS}_{\mathrm{N} 20}$ alone or the lasting decrease in MEP amplitude induced by $\mathrm{PAS}_{\mathrm{N} 20-5}$ alone.

as measured with paired-pulse TMS remained unchanged (Ridding and Taylor, 2001; Stefan et al., 2002). The PAS-induced changes in MEP amplitude last at least $60 \mathrm{~min}$, are input specific, and are blocked by an NMDA receptor antagonist (Stefan et al., 2000, 2002; Ridding and Taylor, 2001; Wolters et al., 2003). These characteristics are consistent with the properties of LTP/D as defined in recordings from single neurons or populations of neurons in slice preparations of animal M1 (Baranyi and Feher, 1981; Hess and Donoghue, 1994; Castro-Alamancos et al., 1995; Hess et al., 1996; Hess and Donoghue, 1996). According to these similarities, the term associative LTP/D-like plasticity was introduced to refer to the PAS-induced changes in MEP amplitude in the human studies (Stefan et al., 2000; Wolters et al., 2003). It should be kept in mind that the term LTP/D-like plasticity should not be equated with LTP/D because, attributable to the noninvasive nature of the PAS studies at the systems level, it cannot be proven definitely that the mechanisms underlying PAS-induced LTP/Dlike plasticity are the same as those underlying LTP/D.

The present findings and one recent study (Wolters et al., 2003) show that the sign of the PAS-induced changes in MEP amplitude depends critically on the temporal order of activation of M1 by the associative stimuli. This property is consistent with spike-timing-dependent plasticity in which LTP occurs if an EPSP is repeatedly paired with a subsequent action potential within a short time window of $<15 \mathrm{msec}$, whereas LTD results if that order is reversed (Markram et al., 1997; Feldman, 2000). However, it is currently unknown whether median nerve stimulation produces an EPSP in neuronal elements in M1, in which an action potential is induced by fTMS. In fact, median nerve stim- 
ulation produces an MEP inhibition at interstimulus intervals around the N20 latency rather than MEP facilitation (Tokimura et al., 2000). Therefore, the exact mechanisms underlying the temporal order effects on LTP/D-like changes in MEP amplitude require additional investigation. This study shows that another important property of the long-lasting changes in MEP amplitude is their stable and reproducible induction in repeated sessions. This was used here to test a given subject repeatedly with the PAS protocol to assess the interactions of motor learning with subsequent PAS-induced plasticity in human M1.

\section{Interactions between motor learning and PAS-induced plasticity}

Although it is known that a distributed network of many cortical areas in addition to $\mathrm{M} 1$, such as the premotor, supplementary motor, dorsolateral prefrontal or parietal cortex, the cerebellum, and various subcortical sites such as the basal ganglia and thalamus may be involved in motor learning (Grafton et al., 1992; Honda et al., 1998; Toni et al., 1998), the present experiments were confined to M1. This was given by the experimental proto$\mathrm{col}$, the primary measure of which was MEP amplitude to test for changes in M1 excitability. In addition, it was justified because previous studies showed that M1 is involved in both motor learning produced by repeated MP (Muellbacher et al., 2001, 2002; Lotze et al., 2003) and PAS-induced plasticity (Stefan et al., 2000; Wolters et al., 2003).

It is less clear, however, which mechanisms underlie motor learning in M1. The major question of this study was to which extent motor learning in human M1 occurs through associative LTP-like plasticity. A causal relationship between LTP and motor learning was often proposed, however, until recently without direct experimental evidence (Donoghue et al., 1996; Sanes and Donoghue, 2000). Other forms of cortical plasticity such as unmasking of preexisting connections (Jacobs and Donoghue, 1991), "awakening" of silent synapses by insertion of postsynaptic AMPA receptors (Malinow et al., 2000), or excitability changes of postsynaptic neurons (Woody et al., 1991) are alternative potential candidate mechanisms for rapidly occurring changes in M1 output organization as a consequence of motor learning. The present experiments were designed to test specifically the interactions of motor learning with associative LTP/Dlike plasticity. Therefore, they do not bear on the possible contribution of any of these other mechanisms to motor learning.

The motor learning task resulted in a rapid increase in maximum peak acceleration of the practiced thumb abduction movement, which was associated with an increase in MEP amplitude of the APB, very similar to previous findings (Muellbacher et al., 2001, 2002). Most likely, the increase in MEP amplitude occurs in M1 because it was not observed when MEPs were elicited by magnetic brainstem stimulation (Muellbacher et al., 2001). The increase in MEP amplitude associated with motor leaning is consistent with the enhancement of layer II/III horizontal cortical connections in rat M1 after motor skill acquisition (RioultPedotti et al., 1998, 2000) and the area increase of practiced M1 representations when tested with intracortical microstimulation in monkeys (Nudo et al., 1996) or rats (Nudo et al., 1996; Kleim et al., 1998).

To which extent LTP participates in this enhancement can be tested by the amount of LTP that is inducible subsequent to motor learning. A requirement for this test is that changes in synaptic strength induced by motor learning and associative LTP take place, at least in part, in the same neuronal network. Although it is presently unknown exactly which neurons of the complex neu- ral network in M1 are responsible for the plasticity effects, it is very likely that motor learning and associative LTP share the same network, because voluntary activation (motor learning) and fTMS (PAS), despite the complex nature of the descending corticospinal volley generated by fTMS, activate largely the same corticospinal neurons (Bawa and Lemon, 1993).

The principal finding of the present study was that motor learning prevented the subsequent induction of associative LTPlike plasticity but enhanced LTD-like plasticity. These interactions between MP and PAS-induced plasticity were specific to the occurrence of motor learning because they were not observed when PAS was preceded by the same amount of a different type of $\mathrm{MP}\left(\mathrm{MP}_{\text {slow }}\right)$, which did not result in motor learning (Fig. 6).

A very similar interaction between motor skill learning and LTP/D induction was found recently in rat M1 (Rioult-Pedotti et al., 1998, 2000). Those authors proposed to explain these findings by a stable synaptic modification range: motor skill learning strengthened synaptic connections up into the range of saturation and, therefore, occluded additional LTP induction. At the same time, motor skill learning increased the range for synaptic weakening (i.e., it amplified the room for LTD induction). Alternatively, it is possible to explain the findings by the BienenstockCooper-Munro (BCM) theory of bidirectional synaptic plasticity, which states that the threshold for LTP/D induction shifts as a function of the history of cortical activity (Bienenstock et al., 1982; Artola et al., 1990; Bear and Kirkwood, 1996). If LTP were involved in a learning process, then the BCM theory predicts an increased threshold for the subsequent induction of LTP and, concomitantly, a decreased threshold for LTD induction. Thus, the probability for the induction of LTD increases when LTP has occurred before. The present experiments showed that motor learning not only reduced subsequent LTP-like plasticity but even resulted in a strong trend toward a reversal to LTD-like plasticity (Fig. 4). This finding is not compatible with saturation of LTP-like plasticity because the maximal interaction effect in that model would be complete occlusion by prior learning (Rioult-Pedotti et al., 2000, their Fig. 3). In contrast, the sliding threshold of LTP/D induction in the BCM theory is compatible with the observed trend of a reversal from LTP-like to LTD-like plasticity and the enhancement of LTD-like plasticity as a consequence of prior motor learning-associated LTP-like plasticity. This intricate relationship between LTLP-like plasticity and learning may explain why it is not possible to learn more than one motor task at a time (Brashers-Krug et al., 1996).

In summary, the modifications of PAS-induced plasticity by prior motor learning in the present experiments are best explained by experience-dependent bidirectional plasticity according to the principles of the BCM theory. Findings support the view that LTP-like mechanisms contribute to motor learning in human cortex.

\section{References}

Artola A, Brocher S, Singer W (1990) Different voltage-dependent thresholds for inducing long-term depression and long-term potentiation in slices of rat visual cortex. Nature 347:69-72.

Baranyi A, Feher O (1981) Synaptic facilitation requires paired activation of convergent pathways in the neocortex. Nature 290:413-415.

Bawa P, Lemon RN (1993) Recruitment of motor units in response to transcranial magnetic stimulation in man. J Physiol (Lond) 471:445-464.

Bear MF, Kirkwood A (1996) Bidirectional plasticity of cortical synapses. In: Cortical plasticity (Frazeli MS, Collingridge GL, eds), pp 191-205. Oxford: BIOS Scientific.

Bienenstock EL, Cooper LN, Munro PW (1982) Theory for the development of neuron selectivity: orientation specificity and binocular interaction in visual cortex. J Neurosci 2:32-48. 
Bliss TV, Collingridge GL (1993) A synaptic model of memory: long-term potentiation in the hippocampus. Nature 361:31-39.

Boroojerdi B, Battaglia F, Muellbacher W, Cohen LG (2001) Mechanisms influencing stimulus-response properties of the human corticospinal system. Clin Neurophysiol 112:931-937.

Brashers-Krug T, Shadmehr R, Bizzi E (1996) Consolidation in human motor memory. Nature 382:252-255.

Castro-Alamancos MA, Donoghue JP, Connors BW (1995) Different forms of synaptic plasticity in somatosensory and motor areas of the neocortex. J Neurosci 15:5324-5333.

Classen J, Liepert J, Wise SP, Hallett M, Cohen LG (1998) Rapid plasticity of human cortical movement representation induced by practice. J Neurophysiol 79:1117-1123.

Donoghue JP, Hess G, Sanes JN (1996) Substrates and mechanisms for learning in motor cortex. In: Acquisition of motor behavior in vertebrates (Bloedel J, Ebner T, Wise SP, eds), pp 363-386. Cambridge, MA: MIT.

Feldman DE (2000) Timing-based LTP and LTD at vertical inputs to layer II/III pyramidal cells in rat barrel cortex. Neuron 27:45-56.

Grafton ST, Mazziotta JC, Presty S, Friston KJ, Frackowiak RS, Phelps ME (1992) Functional anatomy of human procedural learning determined with regional cerebral blood flow and PET. J Neurosci 12:2542-2548.

Hess G, Donoghue JP (1994) Long-term potentiation of horizontal connections provides a mechanism to reorganize cortical motor maps. J Neurophysiol 71:2543-2547.

Hess G, Donoghue JP (1996) Long-term depression of horizontal connections in rat motor cortex. Eur J Neurosci 8:658-665.

Hess G, Aizenman CD, Donoghue JP (1996) Conditions for the induction of long-term potentiation in layer II/III horizontal connections of the rat motor cortex. J Neurophysiol 75:1765-1778.

Honda M, Deiber MP, Ibanez V, Pascual-Leone A, Zhuang P, Hallett M (1998) Dynamic cortical involvement in implicit and explicit motor sequence learning. A PET study. Brain 121:2159-2173.

Jacobs KM, Donoghue JP (1991) Reshaping the cortical motor map by unmasking latent intracortical connections. Science 251:944-947.

Kleim JA, Barbay S, Nudo RJ (1998) Functional reorganization of the rat motor cortex following motor skill learning. J Neurophysiol 80:3321-3325.

Liepert J, Terborg C, Weiller C (1999) Motor plasticity induced by synchronized thumb and foot movements. Exp Brain Res 125:435-439.

Lotze M, Braun C, Birbaumer N, Anders S, Cohen LG (2003) Motor learning elicited by voluntary drive. Brain 126:866-872.

Malinow R, Mainen ZF, Hayashi Y (2000) LTP mechanisms: from silence to four-lane traffic. Curr Opin Neurobiol 10:352-357.

Markram H, Lubke J, Frotscher M, Sakmann B (1997) Regulation of synaptic efficacy by coincidence of postsynaptic APs and EPSPs. Science 275:213-215.
Muellbacher W, Ziemann U, Boroojerdi B, Cohen LG, Hallett M (2001) Role of the human motor cortex in rapid motor learning. Exp Brain Res 136:431-438.

Muellbacher W, Ziemann U, Wissel J, Dang N, Kofler M, Facchini S, Boroojerdi B, Poewe W, Hallett M (2002) Early consolidation in human primary motor cortex. Nature 415:640-644.

Nudo RJ, Milliken GW, Jenkins WM, Merzenich MM (1996) Usedependent alterations of movement representations in primary motor cortex of adult squirrel monkeys. J Neurosci 16:785-807.

Pascual-Leone A, Nguyet D, Cohen LG, Brasil-Neto JP, Cammarota A, Hallett M (1995) Modulation of muscle responses evoked by transcranial magnetic stimulation during the acquisition of new fine motor skills J Neurophysiol 74:1037-1045.

Ridding MC, Taylor JL (2001) Mechanisms of motor-evoked potential facilitation following prolonged dual peripheral and central stimulation in humans. J Physiol (Lond) 537:623-631.

Rioult-Pedotti MS, Friedman D, Hess G, Donoghue JP (1998) Strengthening of horizontal cortical connections following skill learning. Nat Neurosci 1:230-234.

Rioult-Pedotti MS, Friedman D, Donoghue JP (2000) Learning-induced LTP in neocortex. Science 290:533-536.

Sakai K, Ugawa Y, Terao Y, Hanajima R, Furabayashi T, Kanazawa I (1997) Preferential activation of different I waves by transcranial magnetic stimulation with a figure-of -eight shaped coil. Exp Brain Res 113:24-32.

Sanes JN, Donoghue JP (2000) Plasticity and primary motor cortex. Annu Rev Neurosci 23:393-415.

Stefan K, Kunesch E, Cohen LG, Benecke R, Classen J (2000) Induction of plasticity in the human motor cortex by paired associative stimulation. Brain 123:572-584.

Stefan K, Kunesch E, Benecke R, Cohen LG, Classen J (2002) Mechanisms of enhancement of human motor cortex excitability induced by interventional paired associative stimulation. J Physiol (Lond) 543:699-708.

Tokimura H, Di Lazzaro V, Tokimura Y, Oliviero A, Profice P, Insola A, Mazzone P, Tonali P, Rothwell JC (2000) Short latency inhibition of human hand motor cortex by somatosensory input from the hand. J Physiol (Lond) 523:503-513.

Toni I, Krams M, Turner R, Passingham RE (1998) The time course of changes during motor sequence learning: a whole-brain fMRI study. NeuroImage 8:50-61.

Wolters A, Sandbrink F, Schlottmann A, Kunesch E, Stefan K, Cohen LG, Benecke R, Classen J (2003) A temporally asymmetric Hebbian rule governing plasticity in the human motor cortex. J Neurophysiol 89:2339-2345.

Woody CD, Gruen E, Birt D (1991) Changes in membrane currents during Pavlovian conditioning of single cortical neurons. Brain Res 539:76-84. 